\title{
Numerical analysis of the influence of underpinning pile affected by overlapping tunnel with shield method
}

\author{
Ping Ou ${ }^{1,}$, Hong Yuan ${ }^{*}, \mathrm{~b}$, Shifeng $\mathrm{Hu}^{1, \mathrm{c}}$ and Xiongfei Yang ${ }^{1, \mathrm{~d}}$ \\ ${ }^{1}$ MOE Key Laboratory of Disaster Forecast and Control in Engineering, Institute of Applied \\ Mechanics, Jinan University, Guangzhou 510632, China. \\ a435595488@qq.com, btyuanhong@jnu.edu.cn, c897838427@qq.com,d176048785@qq.com
}

\begin{abstract}
Keywords: overlapping tunnel; shield construction; underpinning pile foundation; settlement Abstract. In order to research the influence of underpinning pile foundation after excavating tunnel with shield construction, the three-dimensional numerical simulation is carried out by using FLAC 3D based on the actual project in Shenzhen. Different finite difference models are established respectively by changing the sequence of overlapping tunnel excavation, the spacing of the overlapped tunnel, the diameter and length of the pile. Then the data of the settlement of the pile foundation are obtained and the results of influences are analyzed.
\end{abstract}

\section{Introduction}

The subway construction in china is still growing. More and more application of all kinds of overlapping tunnel were built. In the process of tunnel excavation, in order to ensure the stability of the original buildings, underpinning pile foundation is an effective way for the original buildings. Peck R B. [1], collecting a large number of land surface settlement value and some practical engineering data, put forward the formation of the horizontal settlement curve that is similar to the normal distribution at first by using the rigorous derivation and analyzing. Mairie and Kimura [2] calculated the surface settlement formula by the centrifugal experiment method. Ghsboussi [3,4] using two-dimensional finite element to simulate the process of shield excavation, obtained the theory of formation deformation. Davis and Poulos [5], analyzing the influence of the surrounding pile foundation after excavating tunnel, observed that the displacement of the soil is relative to the elastic modulus of the soil and the relationship between the pile and soil. Linwang Ruan [6] simulated the process of shield tunnel excavation by the three-dimensional finite element method, and got the influence value of the deformation and stress of the pile. Lee [7] analyzed the influence of the two-way consolidation of the existing bearing pile foundation in the process of tunnel excavation by finite element method. Hongbo Zhang [8], viewing the constitutive model of soil as Drucker-Prager model, using numerical analysis method to establish a three-dimensional finite element model of the pile, analyzed the influence of the stress and settlement caused by excavating. At present, there are few analysis of the influence of underpinning pile foundation after excavating overlapping tunnel. And it is mainly aimed at the study of the influence of ground surface settlement. There is few research on the influence of the pile foundation. In this paper, different finite difference models are established respectively by changing the sequence of overlapping tunnel excavation, the spacing of the overlapped tunnel, the diameter and length of the pile. Then the data of the settlement of the pile foundation are obtained and the results of influences are analyzed.

\section{Engineering Survey}

During the planning of Shenzhen Metro, considering the run line layout and the economy, pile foundations were built near the tunnel, then overlapping tunnels instead of common double parallel tunnels are excavated.

According to the literature data [9], the details of each soil layer is shown in Table 1. 
Table 1 Physical parameters of soil layer

\begin{tabular}{lccccc}
\hline \multicolumn{1}{c}{ Name of soil layer } & $\begin{array}{c}\text { Thickness } \\
{[\mathrm{m}]}\end{array}$ & $\begin{array}{c}\text { Bulk modulus } \\
{[\mathrm{K} / \mathrm{MPa}]}\end{array}$ & $\begin{array}{c}\text { Shear modulus } \\
{[\mathrm{G} / \mathrm{MPa}]}\end{array}$ & $\begin{array}{c}\text { Internal friction } \\
\text { angle }\left[{ }^{\circ}\right]\end{array}$ & $\begin{array}{c}\text { Cohesion } \\
{[\mathrm{KPa}]}\end{array}$ \\
\hline $\begin{array}{l}\text { Artificial fill } \\
\text { Powder of cohesive }\end{array}$ & 8 & 10.5 & 8.2 & 26.5 & 29 \\
soil & 4 & 9.61 & 3.68 & 7.1 & 6 \\
Weathered layer & 5 & 12 & & & 37 \\
Strong weathered layer & 6 & 34.48 & 24.79 & 29.7 & 48 \\
Weathered layer & 27 & 80 & 80 & 40 & 50 \\
\hline
\end{tabular}

\section{Model of establishment}

Model assumptions. In order to facilitate the calculation, we do some basic simplification:

(1) The constitutive model of soil is the Kulun (Mohr-Coulomb) model, and the constitutive model of pile foundation is isotropic elastic model.

(2) The soil is homogeneous and have no pore water pressure.

(3) Soil consolidation and secondary consolidation settlement are ignored.

(4) The friction of shield to the soil, the connection reduction effect of the lining, grouting pressure and other construction results are ignored.

(5) The concrete lining and the grouting layer are uniform linear elastic model.

(6) The distribution of pile foundation is symmetrical.

Model of pile and soil. This project is double holes overlapped shield excavation. The distance between the top of the center of the tunnel and the ground is $17 \mathrm{~m}$, and the distance between the bottom of the center of the tunnel and the ground is $29 \mathrm{~m}$. The outer diameter of tunnel is 6 meters, and the inter diameter is $5.4 \mathrm{~m}$. The distance between the two tunnels is one time of the diameter of tunnel. In the process of excavation, the excavation of the line 1 is carried out firstly, and then the line 2 is excavated.

The pile cap is made by C40 concrete. The size of section is $2000 \mathrm{~mm} \times 2000 \mathrm{~mm}$. The diameter of the pile foundation is $1 \mathrm{~m}$. Pile is made by C30 concrete. The length of pile meet the three principles generally [10]:

(1) It needs to meet the bearing capacity of pile foundation.

(2) The bottom of the pile foundation should be not less than $0.5 \mathrm{~m}$ in the weathering layer. If the strong weathered layer is thick, the length of pile refers to principle 1 and 3 .

(3) The bottom of the pile foundation can't be 1 meter higher than the lowest point of the tunnel in order to prevent pile foundation from damaging by structure of the tunnel.

The length of the pile foundation is $26 \mathrm{~m}$, which ensures that the closest distance between the pile and the tunnel should be more than $1 \mathrm{~m}$. The platform needs to span the tunnel, the lateral length of the pile platform is $14 \mathrm{~m}$, and the pile spacing is $12 \mathrm{~m}$. Load, which is $\mathrm{p}=252 \mathrm{t}$, directly load in the center of the beam vertically. The parameters of pile are shown in table 2 .

Table 2 Parameters of pile foundation

\begin{tabular}{cccccc}
\hline Structure & Material & $\begin{array}{c}\text { Bulk modulus } \\
{[\mathrm{K} / \mathrm{MPa}]}\end{array}$ & $\begin{array}{c}\text { Shear } \\
\text { modulus[G/MPa }]\end{array}$ & $\begin{array}{c}\text { Poisson ratio } \\
{[\mathrm{v}]}\end{array}$ & $\begin{array}{c}\text { Density } \\
{\left[\mathrm{KG} / \mathrm{m}^{3}\right]}\end{array}$ \\
\hline Beam & $\mathrm{C} 40$ & $2.7 \mathrm{e} 4$ & $1.6 \mathrm{e} 4$ & 0.3 & 2750 \\
$\begin{array}{c}\text { Underpinning } \\
\text { pile }\end{array}$ & $\mathrm{C} 30$ & $2.5 \mathrm{e} 4$ & $1.5 \mathrm{e} 4$ & 0.3 & 2500 \\
\hline
\end{tabular}

According to the layered strata, the stratum model is divided into 5 layers. The vertical and horizontal dimension is $50 \mathrm{~m}$. The depth of the soil layer is $50 \mathrm{~m}$. The geometric model of soil layer is established by FLAC 3D and the region of the whole model is divided into 81755 grid node and 80556 grid. 
Boundary conditions:

The boundary of the model at the bottom and the surrounding is set to the simply supported boundary by using the command 'fix'.

\section{Results of simulation}

Initial simulation. The sequence of construction is up-to-down. In the process of simulation, it obtains vertical displacement contours after excavating upper tunnel and double holes tunnel respectively.

Verification of excavation simulation. The simulation value is compared with the Peck modified formula method[11] in order to verify the rationality of the influence of settlement on the ground surface after excavating the overlapped tunnel.

Superposition principle: the influence of surface caused by two different deep tunnel excavation is the sum of their respective effects.

Surface settlement caused by overlapping tunnels is:

$$
W(x, y) \approx W_{1}(x, y)+W_{2}(x, y)
$$

Peck correction formula is:

$$
\begin{aligned}
& S_{(1,2)}=S_{1 \max } \exp \left(-\frac{x^{2}}{2 i_{1}^{2}}\right)+S_{2 \max } \exp \left(-\frac{x^{2}}{2 i_{2}^{2}}\right) \\
& S_{1 \max }=\frac{V_{i 1}}{i_{1} \sqrt{2 \pi}} \approx \frac{V_{i 1}}{2.5 i_{1}} \\
& S_{2 \max }=\frac{V_{i 2}}{i_{2} \sqrt{2 \pi}} \approx \frac{V_{i 2}}{2.5 i_{1}} \\
& i=\frac{Z}{\sqrt{2 \pi} t g\left(45^{\circ}-\frac{\phi}{2}\right)} \\
& V_{i}=V_{L} \pi R^{2}
\end{aligned}
$$

The horizontal ground surface settlement caused by excavating the single tunnel and overlapped tunnel is obtained by the formula 2 . The theoretical value is compared with the simulation value, and the results are obtained in Figure 1.
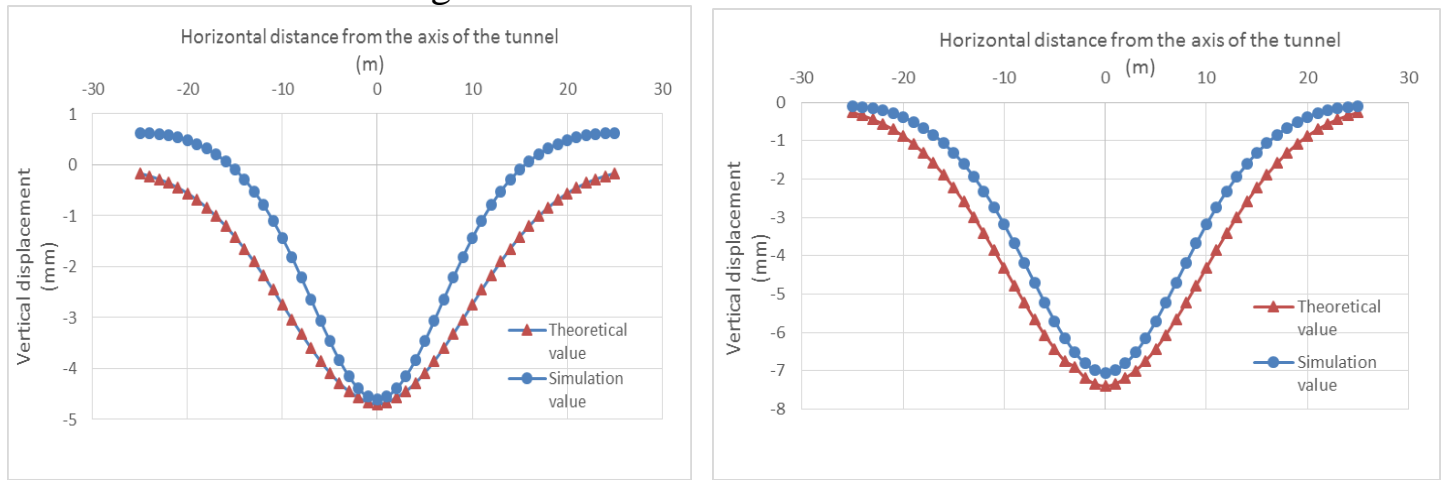

Fig. 1 Lateral surface settlement after excavating 
The settlement trend is very consistent. The sum of squares of difference of the 51 final settling values is $29.7 \mathrm{~mm}$. The average difference of each point is only $0.57 \mathrm{~mm}$. So it can be considered that the formation model is relatively reasonable.

The settlement standard of underpinning pile foundation should be controlled in $+8 \mathrm{~mm}$ (- means rise, + means sink). The maximum settlement of pile foundation is $5.8 \mathrm{~mm}$, the settlement of pile foundation is similar between the modeling values and the measured values. It means the pile and soil model is relatively reasonable.

\section{Analysis of influence factors of displacement of pile foundation}

Influence of underpinning pile affected by sequence of excavation. The horizontal displacement and settlement of underpinning pile foundation are obtained affected by different sequence of excavation simulation, as shown in figure 2 .
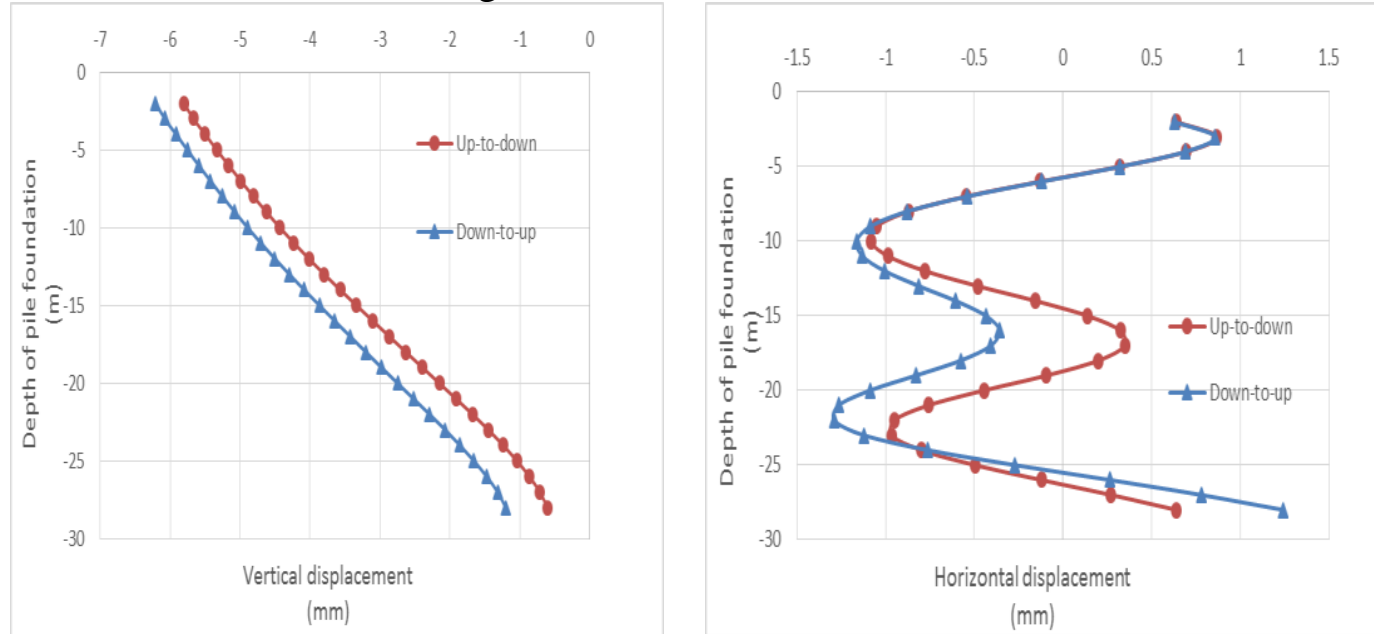

Fig. 2 Influence of displacement of underpinning pile affected by sequence of excavation

The settlement values of different sequences are quite different. The settlement caused by the sequence of up-to-down is smaller, and the variation trend of horizontal displacement is relatively smooth. Above all, the sequence of up-to-down is better than reverse order.

Influence of underpinning pile affected by different spacing of tunnel. The position of the upper tunnel is not moved, and the distance between the two tunnels is one and a half times of the diameter of tunnel. The settlement and horizontal displacement of the pile foundation are obtained, as shown in figure 3 .
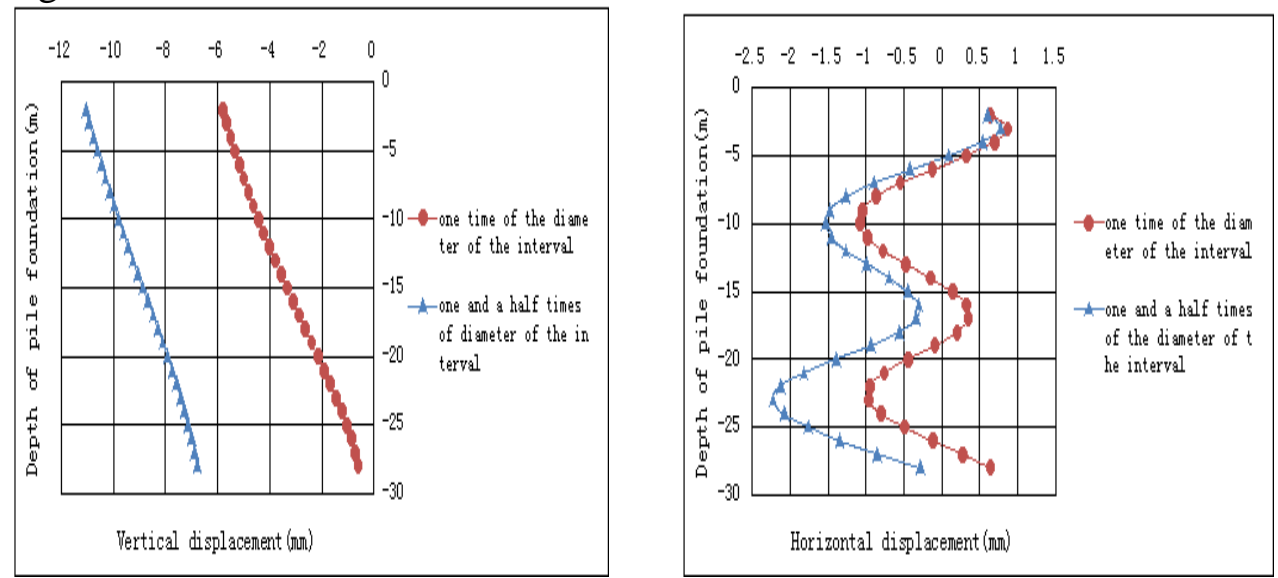

Fig. 3 Influence of displacement of underpinning pile affected by spacing of tunnel

When the distance is 1.5D ( $\mathrm{D}$ means diameter of tunnel), the settlement and horizontal displacement of pile foundation are almost 1 time larger than that of 1D. In pile foundation 
technology, the bottom of the pile foundation is generally not higher $1 \mathrm{~m}$ than the lowest point of the tunnel.

Influence of underpinning pile affected by length of pile foundation. The model of different length of pile is established respectively and the settlement and horizontal displacement of pile foundation are obtained, as shown in figure 4.
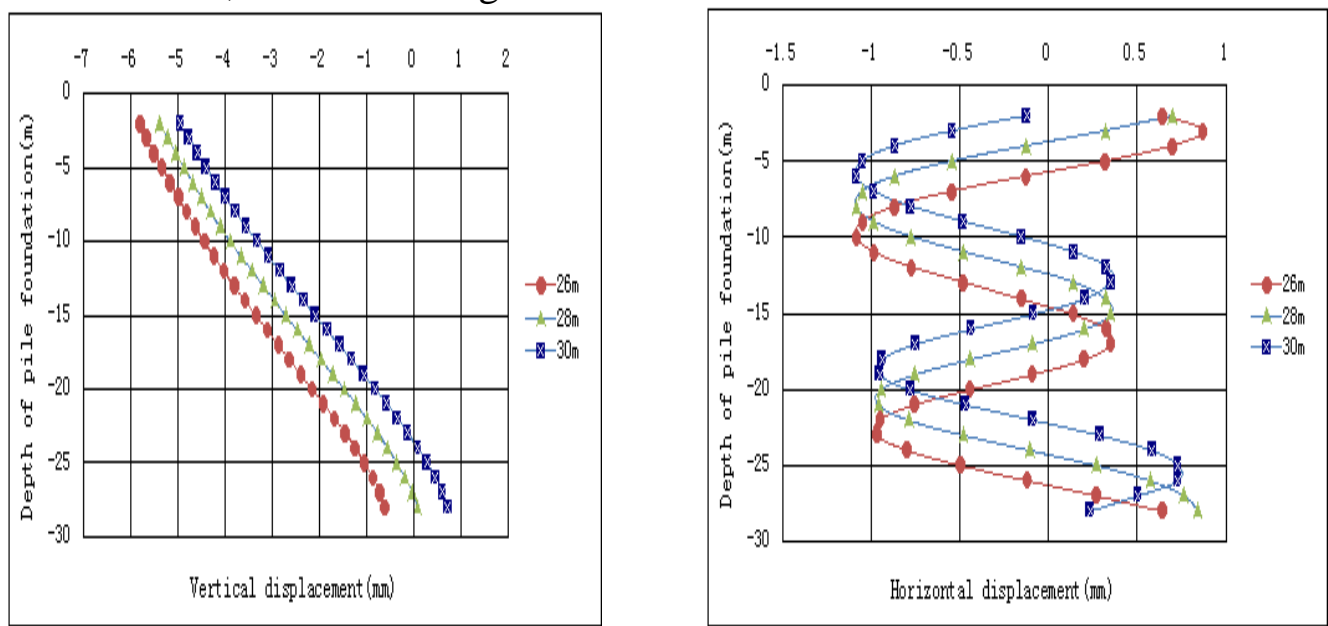

Fig. 4 Influence of displacement of underpinning pile affected by length of pile

The settlement of pile foundation is smaller as the length of pile foundation increase, but the horizontal displacement is not significant difference. When the bottom of the pile foundation is in the hard soil layer, the increasing of length of the pile will help to reduce the settlement.

Influence of underpinning pile affected by diameter of pile foundation. The model of different diameter of pile is established respectively and the settlement and horizontal displacement of pile foundation are obtained, as shown in figure 5 .
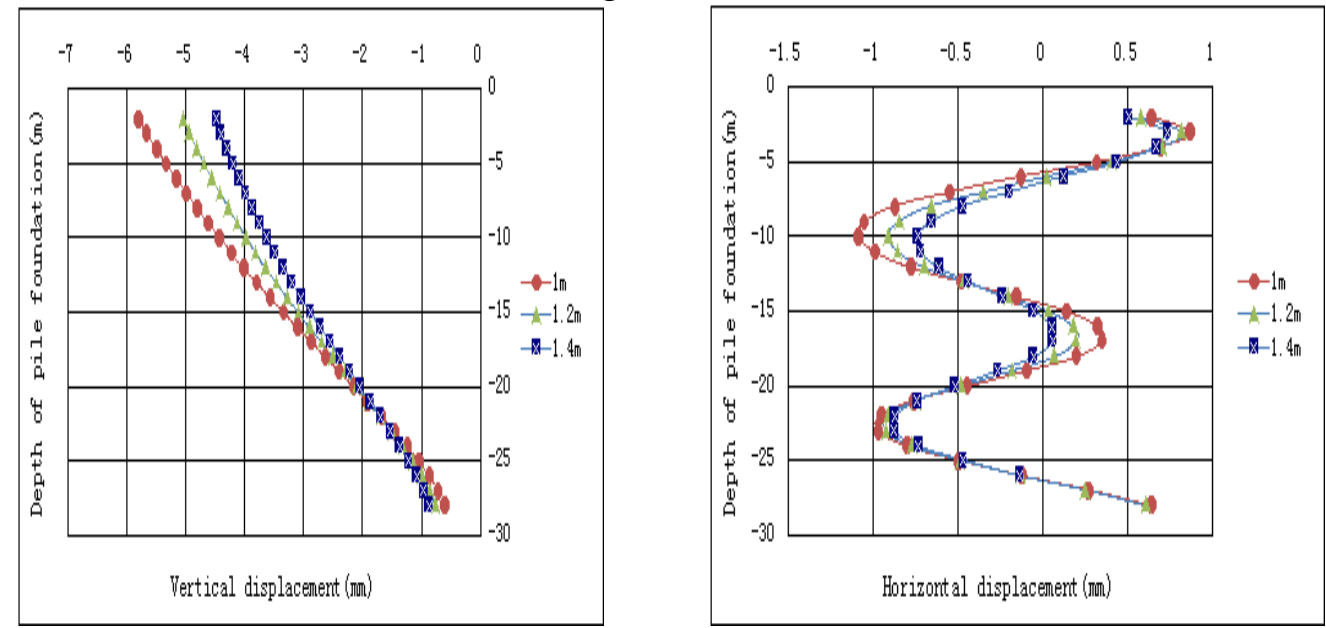

Fig. 5 Influence of displacement of underpinning pile affected by diameter of pile

The settlement and horizontal displacement of pile foundation are smaller as the diameter of pile increase. Overall, the bigger diameter of pile is, the smaller displacement and settlement are.

\section{Conclusions}

Through numerical simulation, the following conclusions are obtained:

(1) The sequence of up-to-down is better than reverse order. The displacement of pile foundation is smaller.

(2) In pile foundation technology, the bottom of the pile foundation is generally not higher $1 \mathrm{~m}$ than the lowest point of the tunnel, which is to prevent the pile foundation from damaging by the tunnel. 
(3) When the bottom of the pile foundation is in the hard soil layer, the increasing of length of the pile will help to reduce the settlement.

(4) The maximum value of horizontal displacement of pile foundation mainly occurs in the vicinity of the vault and the arch of tunnel where the displacement have a big change.

\section{Acknowledgements}

The authors gratefully acknowledge the financial support provided by the Science and Technology Scheme of Guangzhou City (No. 1563000451), the Science and Technology Scheme of Guangdong Province (No. 2012A030200003), and the National Natural Science Foundation of China (No. 11032005).

\section{References}

[1] Peck R B. Deep excavations and tunnelling in soft ground[C]//Proc. 7th int. conf. on SMFE. 1969: 225-290.

[2] Kimura T, Mair R J. Centrifugal testing of model tunnels in soft clay[C]//Proceedings of the 10th international conference on soil mechanics and foundation engineering. ISSMFE: International Society for Soil Mechanics and Foundation Engineering, 1981: 319-322.

[3] Ghaboussi J, Hansmire W H, Parker H W, et al. Finite Element Simulation of Tunneling over Subways[J]. Journal of Geotechnical Engineering, 1983, 109(3):318-334.

[4] Ghaboussi J, Ranken R E, Karshenas M. Analysis of subsidence over soft ground tunnels[J]. Evaluation and Prediction of Subsidence, 1978, 109(3): 182-196.

[5] Poulos, H. G., Davis, E. H. (1980). Pile foundation analysis and design[M]. Wiley, New York.

[6] Linwang Ruan. Two-dimensional finite element analysis of the influence of soft ground shield tunneling on adjacent soil and pile[D]. Shanghai: Tongji Department of geotechnical engineering 1997 (in Chinese).

[7] Lee $\mathrm{G} \mathrm{T} \mathrm{K,} \mathrm{Ng} \mathrm{C} \mathrm{W} \mathrm{W.} \mathrm{Effects} \mathrm{of} \mathrm{advancing} \mathrm{open} \mathrm{face} \mathrm{tunneling} \mathrm{on} \mathrm{an} \mathrm{existing} \mathrm{loaded} \mathrm{pile[J].}$ Journal of Geotechnical and Geoenvironmental Engineering, 2005, 131(2): 193-201.

[8] Hongbo Zhang, Maosong Huang. Construction mechanics analysis of shallow tunnel crossing building pile foundation [J]. Journal of underground space and engineering, 2005. 04, Vol. 1(No. 2) (in Chinese).

[9] Jun Huang, Dingli Zhang. Numerical simulation of the deformation of overlying strata on Metro overlapping tunnel[J]. Journal of rock mechanics and Engineering, 2005.06, Vol. 23(No. 1):2176- 2182 (in Chinese).

[10] Shijun Wang. Technology application of pile underpinning in Guangzhou metro line three[J]. Construction Technique, 2006. 06, Vol. 35(No. 6): 49-52 (in Chinese).

[11] Shuai Zhao. Study on the ground settlement law affected by close overlapped tunnel in Qingdao city [D]. Master's degree thesis of Shandong University of Science and Technology, 2008, 5 (in Chinese). 